'Breeding for Success: Diversity in Action' C.F. Mercer (ed).

\title{
At the root of it all: a QTL analysis of root distribution in perennial ryegrass
}

\author{
MJ Faville $^{1}$, JR Crush $^{2}$ and HS Easton ${ }^{1}$ \\ ${ }^{1}$ AgResearch Ltd., Grasslands Research Centre, PB 11008, Palmerston North, New \\ Zealand . \\ ${ }^{2}$ AgResearch Ltd., Ruakura Research Centre, PB 3123, Hamilton, New Zealand.
}

Abstract. Perennial ryegrass is the key forage component in pasture systems in New Zealand, Australia and Europe, and is typically characterised by a shallow root system. Breeding for a deeper root profile is desirable, as root depth influences drought tolerance, anchoring of plants against removal by grazing animals, and nutrient capture. Difficulties associated with conventional selection for root traits may be alleviated by developing marker-assisted selection (MAS) tools, the first stage of which is the identification of quantitative trait loci (QTL). The objective here was to detect QTL influencing vertical root distribution in ryegrass. A root distribution ratio, $R T D$, was measured in a replicated mapping population $(n=188)$ grown in sand-filled tubes. Six QTL for RTD were detected on five linkage groups, individually accounting for 4 $18 \%$ of phenotypic variation in this trait. In silico comparative analysis indicated colinearity between ryegrass RTD QTL and QTL for root traits in the rice genome. To enrich marker density specifically at RTD QTL, comparative information was used to develop sequence-tagged site (STS) markers from rice genome-aligned ryegrass expressed sequence tags, with, thus far, STS markers ppt014 and ppt019 successfully targeted to QTL qRTD-3.2 and qRTD-6, respectively. This investigation represents the first step towards developing a MAS strategy for improved root distribution and, subsequently, perennial ryegrass cultivars with superior persistence and environmental performance.

\section{Introduction}

Perennial ryegrass (Lolium perenne L.) is the dominant pasture species grown in pastoral systems in New Zealand, Australia and Europe. Persistence may be improved though enhanced drought tolerance and resistance to whole plant removal ("pulling") by grazing animals, both of which are influenced by root system architecture. Root mass in ryegrass is typically shallow, with approximately $80 \%$ of the roots concentrated in the top 10-15 cm of soil (Troughton 1957). A root profile penetrating deeper into the soil is expected to enhance drought tolerance by improving the ability of the plant to access water at depth (Huang et al. 1997), and is a factor in anchoring the plant against "pulling" (Thom et al. 2003). In addition to persistence, increased root density deeper in the soil profile may enhance the interception of leached nitrate (Bowman et al. 1998).

Heritable differences between ryegrass families in root system size and root/shoot ratio have been established (Bonos et al. 2004, Crush et al. 2006), indicating potential to breed for deeper root systems in this species. The relative difficulty associated with conventional selection for root traits may be mitigated by a markerassisted selection (MAS) approach, with molecular marker assays employed as an adjunct to conventional selection. The merit of such a strategy has seen considerable effort in QTL (quantitative trait locus) discovery for root traits in major crops including maize (Tuberosa et al. 2003) and rice (Price and Tomos 1997, Li et al. 2005), and introgression of QTL for root traits using MAS has been demonstrated with some success in rice (Shen et al. 2001, Steele et al. 2005). 
Our objective was to use QTL analysis to identify genetic factors influencing vertical root distribution in perennial ryegrass, using data from a mapping population grown in a root tube system. This represents the first step towards developing a MAS strategy for improved root system characteristics, and thereby enhanced persistence and environmental performance, in perennial ryegrass.

\section{Materials and methods}

Population

Mapping population IxS is the $\mathrm{F}_{1}$ full-sibling progeny $(\mathrm{n}=198)$ of a pair cross between heterozygous parents from cultivars 'Grasslands Impact' and 'Grasslands Samson'. Measurement of vertical root distribution

Single tillers from three clonal replicates of each $F_{1}$ and parental genotype were planted in sand-filled $1 \mathrm{~m}$ deep x $0.09 \mathrm{~m}$ wide rigid plastic tubes, and arranged in a randomised complete block design. Tubes were kept in a temperature-controlled glasshouse and irrigated daily with low-ionic strength nutrient solution (Crush et al. 2005). After 60 days growth, sand profiles were divided into 11 depth increments $(0-5 \mathrm{~cm}, 5-10 \mathrm{~cm}$, and $10 \mathrm{~cm}$ thereafter), washed, and roots dried and weighed. Data used for QTL analysis were $R T D$, the ratio of root dry weight in the $10-20 \mathrm{~cm}$ depth/ root dry weight in the 0 $10 \mathrm{~cm}$ depth.

Genetic linkage analysis and comparative mapping

A genetic linkage map was constructed using JoinMap 3.0 (http://www.kyazma.nl/), with 165 EST-SSR markers (simple sequence repeat markers derived from expressed sequence tags) for population IxS. Marker PCR and genotyping were conducted as in Faville et al. (2004). The macro-syntenic relationship of the ryegrass genome map to the physical rice genome was established in silico, as described previously (Faville 2005).

QTL analysis

QTL analysis of RTD in population IxS was conducted using MQM in MapQTL 4.0 (http://www.kyazma.nl/). Permutation testing $(\mathrm{n}=1000)$ established a logarithm-of-odds (LOD) threshold of 3.42 to declare a QTL at genome-wide significance of $\alpha=0.05$. STS marker development and mapping

Sequence-tagged site (STS) markers were developed from perennial ryegrass ESTs aligned by GMAP (Wu and Watanabe 2005) to positions in the TIGR rice pseudomolecule assembly proximal to rice markers for root trait QTL (root dry weight, root weight, rooting depth, root length, and deep root dry weight) identified at Gramene (http://www.gramene.org). STS primers (18-24 bp long, targeted product length 120 380 bp) were designed with Primer 3 (Rozen and Skaletsky 1998). Primer synthesis, PCR and genotypic analysis were performed as for EST-SSR analysis.

\section{Results and discussion}

Distribution of root mass in the IxS population was consistent with previous observations for perennial ryegrass (Troughton 1957, Crush et al. 2005), with approximately $70 \%$ of root dry weight in the top $20 \mathrm{~cm}$ of the root tubes after 60 days growth (data not presented). Mean RTD values were normally distributed (Figure 1), ranging from 0.138 to 1.010 across 190 genotypes (overall mean $=0.470$ ). Values of 0.245 and 0.313 were obtained for the 'Impact' and 'Samson' parents, respectively, and ANOVA confirmed significant differences in RTD amongst genotypes $(\mathrm{p}<0.0001)$. 


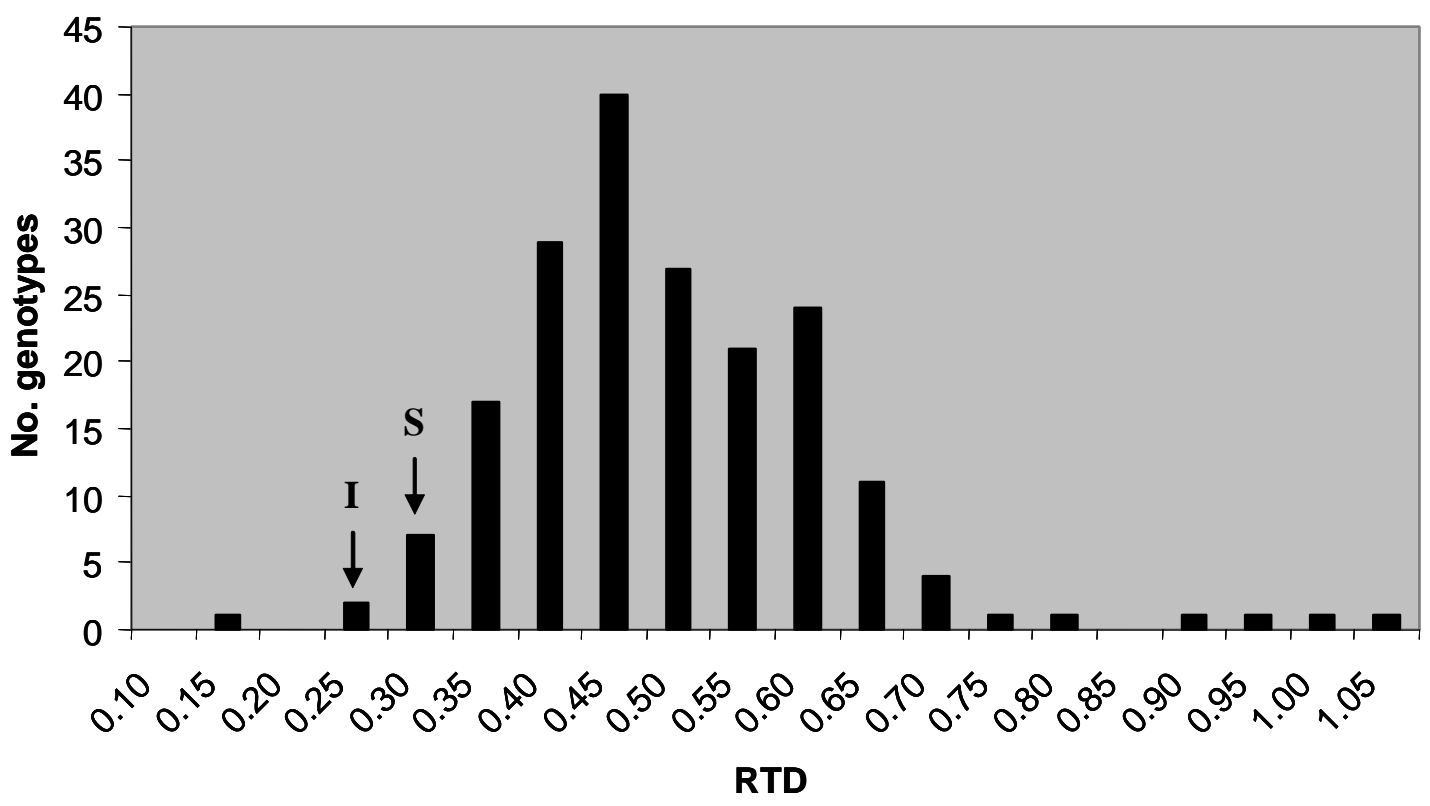

Figure 1. Distribution of mean RTD values (ratio of root dry weight in the $10-20 \mathrm{~cm}$ depth/ root dry weight in the $0-10 \mathrm{~cm}$ depth) amongst $188 \mathrm{IxS}$ population genotypes, plus values for 'Impact' (I) and 'Samson' (S) parents indicated by arrows.

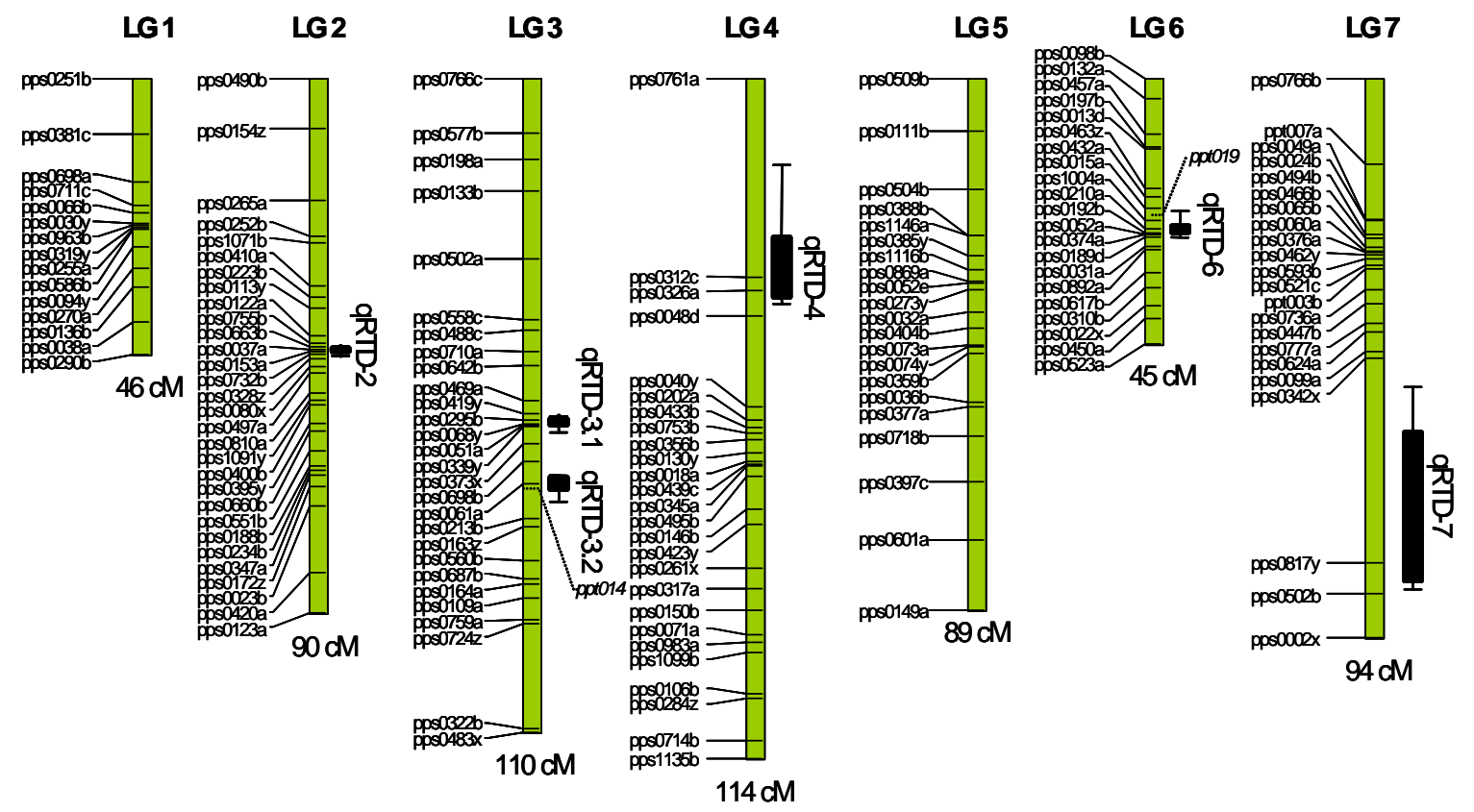

Figure 2. Perennial ryegrass genetic linkage map showing QTL (qRTD-2 to qRTD-7) detected for the root distribution trait RTD. Linkage groups LG 1 to LG7 show marker names at left of bars, QTL positions at right of the bars, and length of linkage groups in centimorgans (cM) at the foot of the bars. The position of STS markers ppt014 and ppt019 are shown at right of LG 3 and LG 6, respectively. 
Table 1. QTL detected for parameter RTD. LOD = LOD score at peak of the QTL, \%PVE = percentage of phenotypic variation explained by QTL. Marker position in rice $=$ rice genome position $(\mathrm{Mb}$, megabase pairs $)$ of putative rice orthologue identified for the ryegrass marker underlying the QTL.

\begin{tabular}{|l|c|c|c|}
\hline QTL & LOD & \%PVE & $\begin{array}{c}\text { Marker position in rice } \\
\text { (chromosome: Mb) }\end{array}$ \\
\hline qRTD-2 & 4.16 & 5.9 & $4: 20.2$ \\
\hline qRTD-3.1 & 8.67 & 17.6 & $1: 22.7$ \\
\hline qRTD-3.2 & 6.51 & 15.1 & $1: 35.6$ \\
\hline qRTD-4 & 4.09 & 6.3 & $3: 0.1$ \\
\hline qRTD-6 & 3.49 & 4.0 & $2: 8.7$ \\
\hline qRTD-7 & 3.69 & 5.6 & $6: 26.2$ \\
\hline
\end{tabular}

Linkage analysis located 164 EST-SSR loci on seven linkage groups (Figure 2) with assignments consistent with other ryegrass maps (Faville et al. 2004). The IxS map is 588 Kosambi cM long, with mean density of $3.6 \mathrm{cM} /$ locus and eight intervals $>10 \mathrm{cM}$. Six genomic regions affecting RDT were identified (Figure 2) by QTL analysis, with single QTL of moderate effect on each of linkage groups LG 2, LG 4, LG 6 and LG 7, and two of large effect on LG 3 (Table 1).

A previously-established comparative framework (Faville 2005) was used to identify rice root trait QTL in positions potentially colinear to ryegrass RTD QTL (Table 2). Particularly close correspondence was identified for qRTD-3.1, -6 and -7. Co-location may reflect conservation of genetic control of root distribution between these species, although this comparison may be influenced by ambiguities associated with genetic linkage analysis.

Anchoring of ryegrass $R T D$ QTL to rice syntenic positions provides access to the rice genome as a template for further marker development. Enrichment of marker density specifically at RTD QTL sites will facilitate development of markers with greater MAS potential and provide a starting point for future QTL fine-mapping activities. STS markers were developed from ryegrass ESTs aligned to rice BAC/PACs carrying root trait QTL markers, with STS markers ppt014 and ppt019 targeted and successfully mapped to the QTL regions occupied by qRTD-3.2 and qRTD-6, respectively (Figure 1). Development of markers targeted to other RTD QTL is in process.

QTL discovery is the first step in the longer process of developing marker assays that may be deployed in a MAS strategy. The next phase of this project will entail testing and further development of QTL-targeted markers, and the analysis and validation of QTL in different genetic backgrounds and environments. This will include establishing the significance of data derived from the root tube system to plant performance in field plots. 
Table 2. Comparison of perennial ryegrass $(\mathrm{RG})$ RTD QTL with rice root trait QTL recorded at Gramene (http://www.gramene.org). RG and rice marker positions refer to the marker underlying the QTL, or nearest flanking marker, and data shown are rice chromosome and position in Mb. Rice QTL map: 1=Aberdeen Bala/Azu QTL 2002, 2=CNHZAU Zh97/Ming63 RI QTL 2002, 3=CNZU Azu/IR1552 RI QTL 2003, 4=Cornell IR64/Azu DH QTL 2001, 5=CTIR CT9993/IR6226 QTL 2000, 6=IGCN, 7=IRRI IR64/Azu DH QTL 2003, 8=TTU IR64/Orufi RI QTL 2003. BAC = bacterial artificial chromosome, $\mathrm{PAC}=\mathrm{P} 1$-derived artificial chromosome.

\begin{tabular}{|l|c|c|cc|cc|}
\hline RG QTL & \multicolumn{2}{|c|}{$\begin{array}{c}\text { RG marker } \\
\text { position in rice }\end{array}$} & $\begin{array}{c}\text { Rice QTL } \\
\text { map }\end{array}$ & \multicolumn{2}{|c|}{$\begin{array}{c}\text { Rice QTL marker } \\
\text { (BAC/PAC) }\end{array}$} & $\begin{array}{c}\text { Rice marker } \\
\text { position }\end{array}$ \\
\hline qRTD-03-2 & $4: 20.2$ & 1,8 & RZ467 (AL606615) & $4: 22.2$ \\
\hline qRTD-03-3.1 & $1: 22.7$ & 8 & bcd134 (AP003075) & $1: 21.6$ \\
\hline qRTD-03-3.2 & $1: 35.6$ & $1,2,3$ & C86 & (AP003235) & $1: 37.7$ \\
\hline qRTD-03-4 & $3: 0.1$ & $3,4,5,7$ & RZ329 & (AC107225) & $3:$ & 3.5 \\
\hline qRTD-03-6 & $2:$ & 8.7 & 6 & RM452 (AP005428) & $2:$ & 9.6 \\
\hline qRTD-03-7 & $6: 26.2$ & 3,5 & RZ140 (AP003517) & $9: 26.4$ \\
\hline
\end{tabular}

\section{Acknowledgements}

Technical assistance was provided by Shirley Nichols, Li Ouyang, Divya Abraham and Alieu Sartie. This work was supported by the New Zealand Foundation for Research, Science and Technology.

\section{References}

Bonos SA, Rush D, Hignight K, Meyer WA (2004) Selection for deep root production in tall fescue and perennial ryegrass. Crop Science 44, 1770-1775.

Bowman DC, Devitt DA, Engelke MC, Rufty Jr., TW (1998) Root architecture affects nitrate leaching from bentgrass turf. Crop Science 38, 1633-1639.

Crush JR, Easton HS, Waller JE (2006) Genetic variation in perennial ryegrass for root profile characteristics. 'Breeding for Success: Diversity in Action' C.F. Mercer (ed). Proceedings of the $13^{\text {th }}$ Australasian Plant Breeding Conference, Christchurch, New Zealand 18-21 April 2006. pp.1073-1077.

Crush JR, Waller JE, Care DA (2005) Root distribution and nitrate interception in eleven temperate forage grasses. Grass and Forage Science 60, 385-392.

Faville MJ (2005) An in silico DNA sequence comparison of the perennial ryegrass and rice genomes. Proceedings of the $4^{\text {th }}$ International Symposium on the Molecular Breeding of Forage and Turf, 181.

Faville MJ, Vecchies AC, Schreiber M, Drayton MC, Hughes LJ, Jones ES, Guthridge KM, Smith KF, Sawbridge T, Spangenberg GC, Bryan GT, Forster JW (2004) Functionally-associated molecular genetic marker map construction in perennial ryegrass (Lolium perenne L.). Theoretical and Applied Genetics 110, 12-32. 
Huang B, Duncan RR, Carrow RN (1997) Drought-resistance mechanisms of seven warm-season turfgrasses under surface soil drying: II. Root aspects. Crop Science 37, 1863-1869.

Li Z, Mu P, Li C, Zhang H, Li Z, Gao Y, Wang G (2005) QTL mapping of root traits in a doubled haploid population from a cross between upland and lowland japonica rice in three environments. Theoretical and Applied Genetics 110, 1244-1252.

Price AH, Tomos AD (1997) Genetic dissection of root growth in rice (Oryza sativa L.). II: mapping quantitative trait loci using molecular markers. Theoretical and Applied Genetics 95, 143-152.

Rozen S, Skaletsky HJ (1998) Primer3. Code available at http://wwwgenome.wi.mit.edu/genome_software/other/primer3.html.

Shen L, Courtois B, McNally KL, Robin S, Li Z (2001) Evaluation of near-isogenic lines of rice introgressed with QTLs for root depth through marker-aided selection. Theoretical and Applied Genetics 103, 75-83.

Steele KA, Price AH, Shashidhar HE, Witcombe JR (2005) Marker-assisted selection to introgress rice QTLs controlling root traits into an Indian upland rice variety. Theoretical and Applied Genetics 112, 208-221.

Thom ER, Burggraaf VT, Watts RJ, Hooper RJ (2003) Relationship of tillering and morphological characteristics of two perennial ryegrass lines to "pulling" when grazed by dairy cows. New Zealand Journal of Agricultural Research 46, 15-25.

Troughton, A. (1957) The underground organs of herbage grasses. Commonwealth Bureau of Pastures and Field Crops 44, 41-42.

Tuberosa R, Salvi S, Sanguineti M-C, Maccaferri M, Giuliani S, Landi P (2003) Searching for quantitative trait loci controlling root traits in maize: A critical appraisal. Plant and Soil 255, 35-54.

Wu TD, Watanabe CK (2005) GMAP: a genomic mapping and alignment programme for mRNA and EST sequences. Bioinformatics 21(9), 1859-1875. 